\title{
Erratum to: A multi-stage genome-wide association study of uterine fibroids in African Americans
}

\author{
Jacklyn N. Hellwege ${ }^{1,2,3} \cdot$ Janina M. Jeff ${ }^{4}$ Lauren A. Wise $^{5,6} \cdot$ C. Scott Gallagher $^{7} \cdot$ Melissa Wellons $^{8,9}$. \\ Katherine E. Hartmann ${ }^{3,9}$ - Sarah F. Jones ${ }^{1,3}$ - Eric S. Torstenson ${ }^{1,2}$ • Scott Dickinson ${ }^{10}$ • Edward A. Ruiz-Narváez ${ }^{6}$. \\ Nadin Rohland $^{7} \cdot$ Alexander Allen $^{7} \cdot$ David Reich $^{7,11,12} \cdot$ Arti Tandon $^{7} \cdot$ Bogdan Pasaniuc $^{13,14} \cdot$ Nicholas Mancuso $^{13}$. \\ Hae Kyung Im $^{10}$ • David A. Hinds ${ }^{15} \cdot$ Julie R. Palmer $^{6} \cdot$ Lynn Rosenberg $^{6} \cdot$ Joshua C. Denny $^{16,17}$. \\ Dan M. Roden ${ }^{2,16,17,18} \cdot$ Elizabeth A. Stewart $^{19}$ - Cynthia C. Morton ${ }^{12,20,21,22} \cdot$ Eimear E. Kenny $^{4}$. \\ Todd L. Edwards ${ }^{1,2,3}$ • Digna R. Velez Edwards ${ }^{2,3,9}$
}

Published online: 4 October 2017

(C) Springer-Verlag GmbH Germany 2017

\section{Erratum to: Hum Genet (2017) 136:1363-1373 DOI 10.1007/s00439-017-1836-1}

The article "A multi-stage genome-wide association study of uterine fibroids in African Americans", written by Jacklyn N. Hellwege, was originally published Online First without open access. After publication in volume 136, issue 10, page 1363-1373 the author decided to opt for Open Choice and to make the article an open access publication. Therefore, the copyright of the article has been changed to (c)

The online version of the original article can be found under doi:10.1007/s00439-017-1836-1.

Digna R. Velez Edwards

digna.r.velez.edwards@ vanderbilt.edu

1 Division of Epidemiology, Department of Medicine, Vanderbilt University Medical Center, Nashville, TN, USA

2 Vanderbilt Genetics Institute, Vanderbilt University Medical Center, Nashville, TN, USA

3 Institute for Medicine and Public Health, Vanderbilt University Medical Center, Nashville, TN, USA

4 Charles Bronfman Institute for Personalized Medicine, Icahn School of Medicine at Mount Sinai, New York, NY, USA

5 Department of Epidemiology, Boston University School of Public Health, Boston, MA, USA

6 Slone Epidemiology Center at Boston University, Boston, MA, USA

7 Department of Genetics, Harvard Medical School, Boston, MA, USA

8 Division of Diabetes, Endocrinology and Metabolism, Department of Medicine, Vanderbilt University Medical Center, Nashville, TN, USA

9 Department of Obstetrics and Gynecology, Vanderbilt University Medical Center, Nashville, TN, USA
The Author(s) 2017 and the article is forthwith distributed under the terms of the Creative Commons Attribution 4.0 International License (http://creativecommons.org/licenses/ by/4.0/), which permits use, duplication, adaptation, distribution and reproduction in any medium or format, as long as you give appropriate credit to the original author(s) and the source, provide a link to the Creative Commons license, and indicate if changes were made.

10 Section of Genetic Medicine, The University of Chicago, Chicago, IL, USA

11 Howard Hughes Medical Institute, Chevy Chase, MD, USA

12 Broad Institute of Harvard and MIT, Cambridge, MA, USA

13 Department of Pathology and Laboratory Medicine, David Geffen School of Medicine, University of California, Los Angeles, Los Angeles, CA, USA

14 Department of Human Genetics, David Geffen School of Medicine, University of California, Los Angeles, Los Angeles, CA, USA

15 23andMe, Inc., Mountain View, CA, USA

16 Department of Biomedical Informatics, Vanderbilt University School of Medicine, Nashville, TN, USA

17 Department of Medicine, Vanderbilt University School of Medicine, Nashville, TN, USA

18 Department of Pharmacology, Vanderbilt University School of Medicine, Nashville, TN, USA

19 Departments of Obstetrics and Gynecology and Surgery, Mayo Clinic and Mayo Clinic School of Medicine, Rochester, MN, USA 
20 University of Manchester Academic Health Science Centre, Manchester, England, UK

21 Department of Obstetrics, Gynecology and Reproductive Biology, Brigham and Women's Hospital, Harvard Medical School, Boston, MA, USA

22 Department of Pathology, Brigham and Women's Hospital, Harvard Medical School, Boston, MA, USA 